\title{
EDITORIAL
}

\section{Slaying the CVD dragon with steroids}

\author{
D.D. Sin, S.F. Van Eeden and S.F.P. Man
}

C ardiovascular diseases (CVD) are a big problem worldwide, accounting for $29 \%$ of all deaths and over 16 million deaths annually [1]. Ischaemic heart disease (IHD) and stroke are by far the most important contributors to total CVD mortality, responsible for $43 \%$ (7.2 million) and 33\% (5.5 million) of all CVD deaths, respectively. At the heart of IHD and stroke is atherosclerosis, characterised by foamy macrophages, cholesterol crystals, smooth muscle proliferation and endothelial dysfunction [2]. The three leading modifiable risk factors for atherosclerosis are hyperlipidaemia, hypertension and tobacco smoking, accounting for $50-75 \%$ of total population attributable risk for CVD mortality [3]. By targeting and treating these risk factors, tremendous improvements in health outcomes from IHD and stroke have been achieved over the past $30 \mathrm{yrs}$, largely owing to population-based reductions in blood pressure, cholesterol, saturated fat intake and cigarette smoking. However, recent disappointing results of torcetrapib (which dramatically raised high-density lipoprotein cholesterol) [4], combination therapy with fenofibrate and simvastatin (which reduced low-density lipoprotein cholesterol) [5] and renin-angiotensin system blockers (which reduced blood pressure) $[6,7]$ for the attenuation of cardiovascular events beyond standard therapy have been sobering; these findings have suggested that we may have reached the limits of risk reduction with these interventions, and raise an urgent call to find novel pathways and targets to further fight the large burden of IHD and stroke worldwide. Unfortunately, there is a dearth of promising compounds in the pipeline and many large pharmaceutical companies, including Pfizer, have abandoned CVD drug development altogether, after a decade of repeated failures [8].

The report in this issue of European Respiratory Journal by OTSUKI et al. [9], showing that inhaled corticosteroids (ICS) are associated with reduced atherosclerosis in patients with asthma, is welcome news for patients with CVD. In this carefully conducted case-control study of 150 patients with asthma and 150 healthy control subjects, OTSUKI et al. [9] showed that patients with asthma in general had lower burden of carotid atherosclerosis, as determined by high-resolution ultrasound B-mode imaging studies demonstrating reduced intimal and medial wall thickness and reduced plaque formation in the carotid arteries, in spite of a higher prevalence

Dept of Medicine, Division of Respirology, The University of British Columbia and Providence Heart and Lung Institute, and The James Hogg Research Center at the Providence Heart and Lung Institute, St. Paul's Hospital, Vancouver, BC, Canada.

CORRESPONDENCE: D.D. Sin, UBC James Hogg Research Center, Providence Heart and Lung Institute, St Paul's Hospital, Room \#368A, 1081 Burrard Street, Vancouver, BC V6Z 1Y6, Canada. E-mail: don.sin@hli.ubc.ca of smoking among asthmatic than control subjects. In this study, the leading risk factors for increased carotid wall thickness were, not surprisingly, older age, dyslipidaemia and male sex, which comprise three of the five Framingham Heart Study risk factors [10]. However, contrary to expectations, the mean daily dose of ICS was also associated with atherosclerosis such that patients who were using the highest doses of ICS had the lowest risk while those using the lowest doses had the highest risk, suggesting that ICS is protective against atherosclerosis [9]. The use of other anti-asthma medications, such as $\beta_{2}$-agonists, leukotriene receptor antagonists and theophylline, made no difference to the risk of atherosclerosis.

The strengths of this study were the relatively large sample size, the direct visual measurement of atherosclerosis, the careful blinding of the ultrasonographer to the clinical status of the patients, and the excellent balance in the baseline demographic characteristics between asthmatic patients and control subjects. However, there were also some shortcomings. First, it was not a randomised controlled trial and, as such, was susceptible to the biases and confounding issues that plague all observational studies [11]. Secondly, it was a cross-sectional study and so lacks the power of a longitudinal study for the assessment of temporal relationships. Thirdly, the patients used different formulations of ICS. To make different compounds comparable, all ICS were converted to the same units by using a conversion factor of 1.25 for budesonide, and 2.0 for fluticasone propionate, hydrofluoroalkane beclomethasone and hydrofluoroalkane ciclesonide. Although this approach is reasonable and practical, it is far from precise, making it possible that residual errors in dose conversion may have driven the results. Fourthly, the potential role of the "asthma phenotype" in CVD pathogenesis is unclear, given that the control group was comprised of healthy subjects. Lastly, and most importantly, on the surface at least, there appears to be no compelling biological rationale for why ICS would reduce the risk of atherosclerosis. Indeed, the traditional teaching has been that steroids accelerate atherosclerosis by exacerbating several well-known coronary risk factors such as hypertension, hyperlipidaemia and glucose intolerance [12]. While this statement is true for oral corticosteroids, perhaps because of the low blood levels, ICS do not appear to share these side-effects.

What then is a plausible mechanism? It is now well established that, along with the traditional risk factors, inflammation plays an active role in the pathogenesis of atherosclerosis [13]. Once the endothelium becomes activated by sheer stress or circulating pro-inflammatory mediators, a local inflammatory reaction occurs in the vessel wall that allows translocation of oxidised low-density lipoprotein and leukocytes that amplify blood 
vessel wall inflammation (fig. 1). This process can be further enhanced by the absence of high-density lipoprotein (which carries the processed cholesterol from the vessels to the liver), or by persistent (systemic) inflammatory stimulus, leading to chronic elevations in plasma C-reactive protein or interleukin-6 levels (fig. 1) [14]. Local application of corticosteroids to damaged endothelium, however, can attenuate the inflammatory process and restore the damaged endothelium. This concept is well supported by animal experiments. For example, VILLA et al. [15] showed that local application of $0.5 \%$ dexamethasone polymer to damaged endothelium prevented neointimal proliferation following balloon vascular injury and restored vascular function in a rat model. In a mouse model, we have shown that, by spraying just one dose of budesonide $\left(2 \mathrm{mg} \cdot \mathrm{kg}^{-1}\right)$ into the trachea of 10-15-week-old mice, we could restore the endothelial function of the aorta, which was lost by intratracheal instillation of lipopolysaccharide [16].
Similar findings have been noted in other animals, including rabbits [17].

This concept also appears to be applicable to humans. In healthy volunteers, 2-week treatment with fluticasone, intranasally and by inhalation, prevented the rise in plasma interleukin-6 that occurred when subjects were exposed to organic dust [18]. ICS also restored endothelial function when given daily for 3 weeks to smokers [19] and in those with chronic obstructive pulmonary disease (COPD) [20]. Most provocatively, in the EUROSCOP (European Respiratory Society study on chronic obstructive pulmonary disease) study, 3-yr treatment with budesonide was associated with a $43 \%$ reduction in ischaemic cardiac events compared with placebo (number needed to treat 44) [21] and in the INSPIRE (Investigating New Standards for Prophylaxis in Reducing Exacerbations) study, the use of ICS/long-acting $\beta_{2}$-agonist

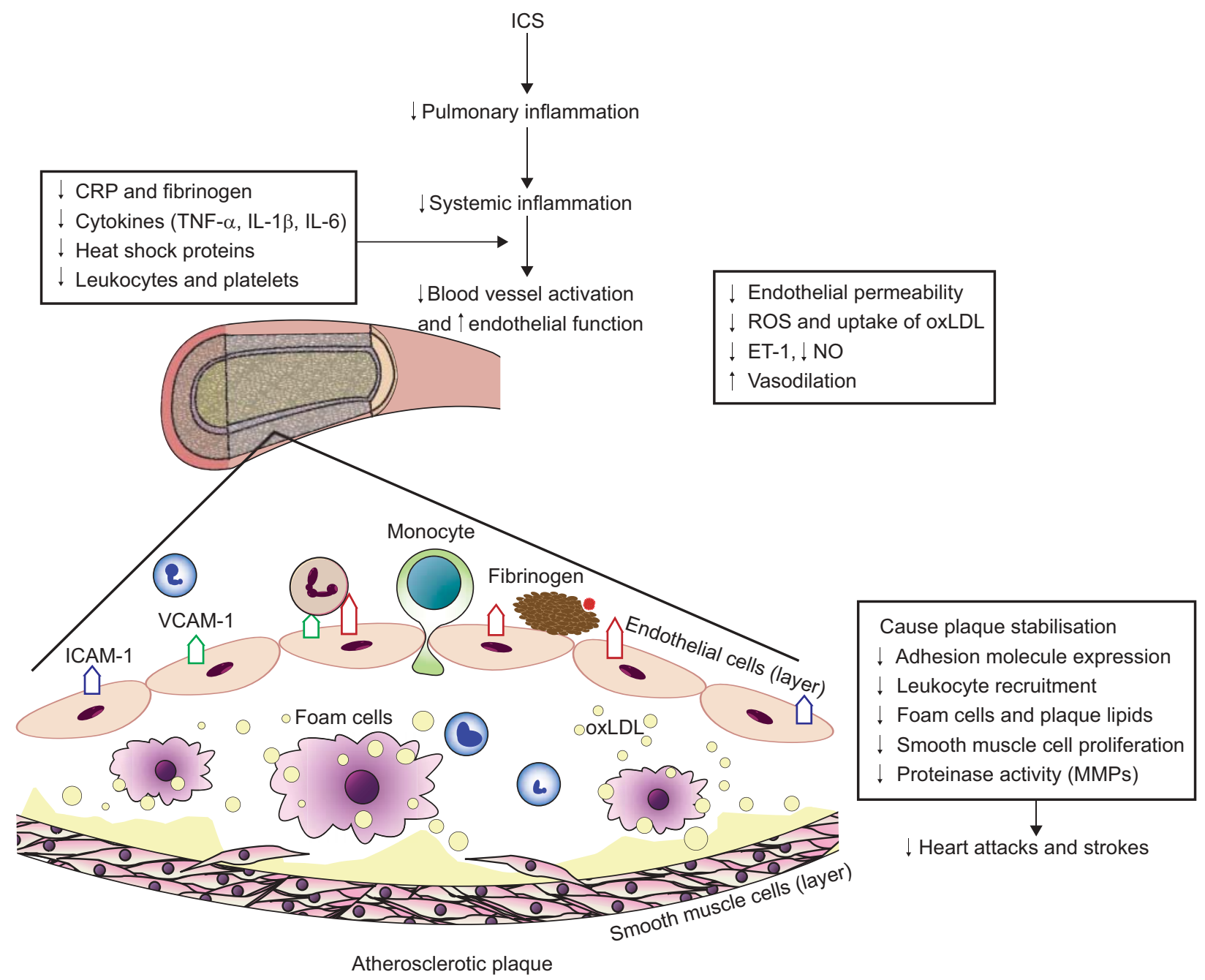

FIGURE 1. Effects of inhaled corticosteroids (ICS) on blood vessels and progression of atherosclerosis. ICS can downregulate the inflammatory process in the lungs, which in turn, reduces the spillover of inflammatory mediators from the lungs into the systemic circulation, dampening the systemic inflammatory process that is persistent in patients with obstructive airways disease. Because systemic inflammation is a co-factor in the genesis of endothelial dysfunction and progression of atherosclerosis, ICS can enhance blood vessel function and reduce the risk of ischaemic events. Critical to this process are mediators such as interleukin (IL)-6, tumour necrosis factor (TNF)- $\alpha$, matrix metalloproteinases (MMPs) and nitric oxide, which may be modulated by ICS. CRP: C-reactive protein; ET-1: endothelin-1; ICAM-1: intercellular adhesion molecule-1; oxLDL: oxidised low-density lipoprotein; ROS: reactive oxygen species; VCAM-1: vascular cell adhesion molecule-1. 
combination resulted in a $31 \%$ reduction in cardiac adverse events and $56 \%$ reduction in total mortality compared with treatment with tiotropium alone, despite no significant differences in lung function or COPD exacerbation rates in the two groups [22]. In the observational study by LEE et al. [23], the use of ICS was associated with a $20 \%$ relative risk reduction for cardiovascular mortality. Collectively, these human and animal data suggest that ICS reduce cardiovascular morbidity and mortality, most likely by reducing complications related to atherosclerosis. While the precise mechanism by which ICS exert this effect has not been fully elucidated, ICS may mitigate the risk of atherosclerosis by reducing local (lung) and systemic inflammation and by reducing endothelial activation, thereby restoring endothelial function, which have been dysregulated by cigarette smoking, infection, inflammation or environmental pollutants (fig. 1).

There is mounting evidence that many of the heart, lung and vascular diseases may have a common pathophysiology. The report by OTSUKI et al. [9] suggests that ICS, which have been used for decades in the treatment of inflammatory airway diseases, may have a salient role in protecting against atherosclerosis. If these data can be confirmed by a large randomised controlled trial, we may be able to at least partially slay the CVD dragon, which, by 2030, will be responsible for one out of every three deaths worldwide (representing 24.2 million deaths per annum) [24], and revolutionise the way in which we treat heart disease.

\section{STATEMENT OF INTEREST}

Statements of interest for all authors can be found at www.erj. ersjournals.com $/ \mathrm{misc} /$ statements.dtl

\section{ACKNOWLEDGEMENTS}

D.D. Sin is a senior scholar with the Michael Smith Foundation for Health Research (MSFHR) and a Canada Research Chair in COPD, and S.F. Van Eeden is a senior scholar with the MSFHR and holds the GlaxoSmithKline Professorship in COPD.

\section{REFERENCES}

1 World Health Organization. Global Strategy on Diet, Physical Activity and Health. 2010. www.who.int/dietphysicalactivity/ publications/facts/cvd/en/ Date last accessed: April 26, 2010. Date last updated: February 19, 2009.

2 Ross R. Atherosclerosis: an inflammatory disease. N Engl J Med 1999; 340: 115-126.

3 Hole DJ, Watt GC, Davey-Smith G, et al. Impaired lung function and mortality risk in men and women: findings from the Renfrew and Paisley prospective population study. BMJ 1996; 313: 711-715.

4 Barter PJ, Caulfield M, Eriksson M, et al. Effects of torcetrapib in patients at high risk for coronary events. N Engl J Med 2007; 357: 2109-2122.

5 ACCORD Study Group, Ginsberg HN, Elam M, et al, Effects of combination therapy in type 2 diabetes mellitus. $N$ Engl J Med 2010; 362: 1563-1567.
6 McMurray JJ, Holman RR, Haffner SM, et al. Effect of valsartan on the incidence of diabetes and cardiovascular events. $N$ Engl J Med, 362: 1477-1490.

7 Yusuf S, Diener HC, Sacco RL, et al. Telmisartan to prevent recurrent stroke and cardiovascular events. N Engl J Med 2008; 359: 1225-1237.

8 Reuters. Pfizer Plans to Abandon Heart Drug Development - WSJ. www.reuters.com/article/idUSN2941302420080930 Date last accessed: May 21, 2010. Date last updated: September 29, 2008.

9 Otsuki M, Miyatake A, Fujita K, et al. Reduced carotid atherosclerosis in asthmatic patients treated with inhaled corticosteroids. Eur Respir J 2010; 36: 503-508.

10 Wilson PW, D'Agostino RB, Levy D, et al. Prediction of coronary heart disease using risk factor categories. Circulation 1998; 97: 1837-1847.

11 Brennan $\mathrm{P}$, Croft $\mathrm{P}$. Interpreting the results of observational research: chance is not such a fine thing. BMJ 1994; 309: 727-730.

12 Nashel DJ. Is atherosclerosis a complication of long-term corticosteroid treatment? Am J Med 1986; 80: 925-929.

13 Hansson GK. Inflammation, atherosclerosis, and coronary artery disease. N Engl J Med 2005; 352: 1685-1695.

14 Danesh J, Kaptoge S, Mann AG, et al. Long-term interleukin-6 levels and subsequent risk of coronary heart disease: two new prospective studies and a systematic review. PLoS Med 2008; 5: e78.

15 Villa AE, Guzman LA, Chen W, et al. Local delivery of dexamethasone for prevention of neointimal proliferation in a rat model of balloon angioplasty. J Clin Invest 1994; 93: 1243-1249.

16 Suda K, Tamagawa E, Wei R, et al. Interleukin-6 translocates from the lungs into the systemic circulation during respiratory infections. Am J Respir Crit Care Med 2009; 179: A1497.

17 Poon M, Gertz SD, Fallon JT, et al. Dexamethasone inhibits macrophage accumulation after balloon arterial injury in cholesterol fed rabbits. Atherosclerosis 2001; 155: 371-380.

18 Ek A, Palmberg L, Larsson K. The effect of fluticasone on the airway inflammatory response to organic dust. Eur Respir J 2004; 24: 587-593.

19 Mendes ES, Horvath G, Rebolledo P, et al. Effect of an inhaled glucocorticoid on endothelial function in healthy smokers. J Appl Physiol 2008; 105: 54-57.

20 Mendes ES, Campos MA, Wanner A. Airway blood flow reactivity in healthy smokers and in ex-smokers with or without COPD Chest 2006; 129: 893-898.

21 Lofdahl CG, Postma DS, Pride NB, et al. Possible protection by inhaled budesonide against ischaemic cardiac events in mild COPD. Eur Respir J 2007; 29: 1115-1119.

22 Wedzicha JA, Calverley PM, Seemungal TA, et al. The prevention of chronic obstructive pulmonary disease exacerbations by salmeterol/fluticasone propionate or tiotropium bromide. Am J Respir Crit Care Med 2008; 177: 19-26.

23 Lee TA, Pickard AS, Au DH, et al. Risk for death associated with medications for recently diagnosed chronic obstructive pulmonary disease. Ann Intern Med 2008; 149: 380-390.

24 The Future. In: Mackay J, Mensah GA. The Atlas of Heart Disease and Stroke. Geneva/Druid Hills, World Health Organization/ Centers for Disease Control and Prevention, 2004. Available from: www.who.int/cardiovascular_diseases/en/cvd_atlas_25_future.pdf 